\title{
Skewness of cloud droplet spectrum and an improved estimation for its relative dispersion
}

\author{
Yu Liu' ${ }^{1}$ Chunsong $\mathrm{Lu}^{2} \cdot$ Weiliang $\mathrm{Li}^{3}$
}

Received: 13 January 2016/Accepted: 9 May 2016/Published online: 20 May 2016

(c) The Author(s) 2016. This article is published with open access at Springerlink.com

\begin{abstract}
The relative dispersion of the cloud droplet spectrum is a very important parameter in describing and modeling cloud microphysical processes. Based on the definition of skewness as well as theoretical and data analyses, a linear fitting relationship $(\alpha=2.91 \varepsilon-0.59)$ between skewness $(\alpha)$ and relative dispersion $(\varepsilon)$ is established and a new method is developed to estimate the relative dispersion of the cloud droplet spectrum. The new method does not depend on any assumption of a particular distribution for the cloud droplet spectrum and has broader applicability than the previous methods. Comparisons of the three methods for the relative dispersion with the observed data supported the following conclusions. (1) The skewness of the cloud droplet spectrum is asymmetrically distributed. An assumption of zero skewness in quantifying the relative dispersion inevitably results in relatively large deviations from the observations. Errors of the estimated relative dispersion due to the omission of the skewness term are not solely related to the skewness, but rather to the product of the skewness and relative dispersion. (2) The use of the assumption that the cloud droplet spectrum takes a gamma distribution is similar to the assumption that the
\end{abstract}

Responsible Editor: S.-W. Kim.

Yu Liu

liuyu@camscma.cn

1 State Key Laboratory of Severe Weather, Key Laboratory of Atmospheric Chemistry of CMA, Chinese Academy of Meteorological Sciences, Beijing 100081, China

2 Key Laboratory for Aerosol-Cloud-Precipitation of China Meteorological Administration, Nanjing University of Information Science and Technology, Nanjing 210044, China

3 Chinese Academy of Meteorological Sciences, Beijing 100081, China skewness is twice the relative dispersion. This leads to a better accuracy in estimating the relative dispersion than that with zero skewness assumption. (3) Comparisons with observations show that the new method is more accurate than the one under gamma distribution assumption and is the best among all the three methods. (4) It is believed that finding a better correlation between the skewness and the relative dispersion would further reduce the deviations for the estimated relative dispersion.

\section{Introduction}

Clouds cover approximately $60 \%$ of the Earth's surface area (Rossow and Schiffer 1999; Deng et al. 2009) and have an important impact on the radiation budget of the earth-atmosphere system including both solar radiation and long-wave radiation components. Clouds are known to be affected by atmospheric dynamical processes and microphysical processes and their variations exhibit a great degree of complexity ( $\mathrm{Lu}$ et al. 2016). It is also believed that clouds are one of the most important sources of uncertainty in climate change, because changes in any of the cloud attributes, for example its amount, spatial distribution, lifetime and optical properties, can certainly alter the atmosphere radiative transfer processes and radiation balance.

The optical properties of clouds are a function of the effective radius $\left(r_{\mathrm{e}}\right)$ of cloud droplets. The cloud optical thickness $(\tau)$ is given by

$\tau=3 H L / 2 \rho_{\mathrm{w}} r_{\mathrm{e}}$,

where $H$ is the thickness of the cloud, $\rho_{\mathrm{w}}$ the water density, and $L$ the cloud liquid water content. Under the two-stream 
approximation of a homogeneous, plane-parallel and nonabsorbing cloud, the albedo of the cloud top $(R)$ is given by (Bohren 1987),

$R=[(1-g) \tau] /[2+(1-g) \tau]$,

where $g$ is the asymmetry factor.

Inspection of Eqs. (1) and (2) reveals that the effective cloud droplet radius affects the cloud optical properties. The effective cloud droplet radius is related to the volume radius $\left(r_{3}\right)$ by a ratio $\left(\beta, r_{\mathrm{e}}=\beta r_{3}\right)$, which in turn is a function of the relative dispersion of the cloud droplet spectrum ( $(\varepsilon)$ (Pontikis and Hicks 1992; Martin et al. 1994; Liu and Daum 2000, 2002).This indicates that the relative dispersion therefore plays a key role in determining the cloud optical properties because of its intrinsic relationship with effective cloud droplet radius. Moreover, $\varepsilon$ also affects the automatic conversion rate from cloud water into rain water (Liu et al. 2006a; Xie et al. 2013), and consequently alters the lifetime of clouds. It is thus evident that the relative dispersion of cloud droplet spectrum $(\varepsilon)$ is a highly important cloud microphysical parameter.

Many studies have investigated the methods for obtaining the relative dispersion of the cloud droplet spectrum. Most of them employed observational data to establish a statistical relationship between the cloud droplet number concentration and the relative dispersion. Some of these statistical relationships showed a positive correlation between them, i.e., $\varepsilon$ increased with the increase of cloud droplet number concentration (CN) (Martin et al. 1994; Hudson and Yum 1997; Miles et al. 2000; Liu and Daum 2002; Liu et al. 2006b), while others revealed a negative correlation (Pawlowska et al. 2006; Ma et al. 2010; Lu et al. 2012). Furthermore, some of these studies have also shown that $\varepsilon$ converges at high cloud droplet number concentration (Zhao et al. 2006; Lu et al. 2007). These inconsistent conclusions thus suggest a complex relationship between the relative dispersion and the cloud droplet number concentration and a possibility that other factors may also play a part in affecting the cloud droplet relative dispersion. In addition to the statistical studies, theoretical models have been developed to estimate the cloud droplet spectral distribution. One such example was to obtain the analytical expression for relative dispersion through simplifying cloud droplets condensation growth equation. However, this approach failed to take into account the effects of the turbulence and collision processes and was only applicable to non-precipitation stratus clouds (Liu et al. 2006b). In summary, the factors controlling and affecting $\varepsilon$ are currently poorly understood.

At present, many weather and climate models adopt the two-moment cloud microphysics scheme to simulate cloud processes by predicting cloud droplet number concentration and the mixing ratio of the hydrometeor composition.
Most of these schemes represent the cloud droplet spectrum using a three-parameter gamma distribution, $n(r)=N r^{\mu} e^{-\lambda r}$, where $n(r)$ is the cloud droplet number concentration per unit of volume and per unit of radius; $r$ is the cloud drop radius; and $N, \mu$, and $\lambda$ are the parameters. $N$ is often referred to as the intercept, $\lambda$ is the slope parameter, and $\mu$ is related to the spectral width and commonly referred to as the shape parameter (Ferrier 1994; Meyers et al. 1997; Cohard and Pinty 2000; Milbrandt and Yau 2005a; Morrison et al. 2005). $N$ and $\lambda$ can be obtained from the cloud droplet number concentration and the hydrometeor mixing ratio, while $\mu$ is assumed to be a constant or obtained using the statistical relationship between the relative dispersion and cloud droplet number concentration $\mu$, $\left(\varepsilon^{2}=\frac{1}{(\mu+1)}\right)$. Because of the lack of a generally valid statistical relationship between the relative dispersion and the cloud number concentration, other methods have been developed to obtain $\mu$ values. For example, a kinetic equation for the cloud droplet spectral shape parameter was proposed and, after some simplifications and under several assumptions, an analytic expression for the shape parameter $\mu$ could be derived. However, this method failed to produce satisfactorily accurate results (Khvorostyanov and Curry 1999a, b). In another study, a triple-parameter cloud microphysics scheme was developed (Milbrandt and Yau 2005b) that adopted the radar reflectivity of six moments as the predicted variable to derive the shape parameter. However, an analytical solution for the shape parameter from this method is infeasible and its requirement of intensive computational power prevents the widespread use of this method in modeling. A cloud microphysics scheme with multiple parameters was recently developed (Kogan and Belochitski 2012) to obtain a relational expression for the high-order moment of cloud droplets using bin modeling. This method requires an increased number of the predicted variables and is computationally intensive, but at the same time offers only limited improvement on accuracy in comparison to the two-parameter scheme.

In a very recent work based on the two-parameter cloud microphysics scheme, Liu and Li (2015) used the cloud droplet condensation growth equation and exploited the features of gamma function to find solutions for obtaining the average radius of cloud droplets and the cloud droplet spectrum shape parameter. Using the average radius, volume radius, and their ratio, the cloud droplet spectrum shape parameter could be obtained first and, subsequently, the relative dispersion and cloud droplet distribution. With this approach, the two-parameter cloud microphysics scheme becomes a triple-parameter cloud microphysics scheme. A primary advantage of this method is that analytical solutions are achievable for the shape parameters 
and the relative dispersion. An application of this method to the WRF model has evidently improved the precipitation modeling to some degree. As mentioned above, this approach has been founded on an assumption of gamma distribution for the cloud droplet spectrum. Although a large number of observations have provided support to this assumption (Mason 1971; Sedunov 1974; Cotton and Anthes 1989; Pruppacher and Klett 1997), some other observations have shown exceptional examples (Deng et al. 2009). Following our previous work, here we seek a general method for obtaining the relative dispersion and at the same time eliminate the assumption of gamma distribution with an aim of widening the application of this method.

\section{Data and evaluation method}

To analyze the skewness of the cloud droplet spectrum and evaluate our proposed method, we use the cloud droplet spectral data from the RACORO experiment (the Routine ARM aerial facility CLOWD Optical Radiative Observations campaign). The RACORO experiment was designed to investigate the boundary layer clouds over land, evaluate the high-resolution models, and improve the retrieval method. The experiment was conducted at Lemont, Oklahoma, USA (Southern Great Plains) from January 22 to June 30, 2009. Boundary layer clouds are frequently observed at the experiment site, and the high-quality surface observation system employed by this research campaign can support simulation studies and facilitate the assessment of various retrieval methods on the ground (Vogelmann et al. 2012). In the RACORO project, research aircraft (Twin Otter) of the research center of interdisciplinary remote control aircraft (CIRPAS) was used to comprehensively observe cloud, aerosols, radiation, and other meteorological elements on multiple vertical levels. The sampling heights were different from one flight to another, largely varying between 500 and $3500 \mathrm{~m}$. In general, the flights were conducted once above the cloud top, once below the cloud base, and three times within the clouds. A cloud and aerosol particle spectrometer (DMTUSA Inc.) was deployed to measure cloud droplet spectrum at a sampling frequency of $10 \mathrm{~Hz}$. This instrument measures the aerosol and cloud droplets within a radius in the $0.29-25 \mu \mathrm{m}$ range, performing 20 interval measurements for the aerosol and cloud particles. Only cloud particles larger than $1 \mu \mathrm{m}$ of average radius are considered in this work and included to calculate the cloud microphysical quantities ( $\mathrm{Lu}$ et al. 2013). The experiment took more than 5 months and 260 flight hours, obtaining a large amount of data for the boundary layer cloud and aerosol properties.
To quantitatively evaluate the estimation methods, two measures are adopted: correlation coefficient between estimates and observations and standard error $(\sigma$, root mean square error). The latter is defined as follows:

$\sigma=\sqrt{\frac{\sum_{i=1}^{n}\left(x_{i}-x\right)^{2}}{n}}$,

where $X_{i}$ is the estimated quantity, $X$ is the observation, and $n$ is the number of samples.

\section{Estimation method for relative dispersion}

The relative dispersion of cloud droplet spectrum is given by (Pontikis and Hicks 1992)

$\varepsilon=\frac{\sigma}{r_{1}}$

where $\sigma$ is the standard deviation of cloud droplet spectrum, $r_{1}$ is the average radius, and $\varepsilon$ reflects the degree of the mean deviation relative to the average radius of the cloud droplet spectrum distribution.

According to the definition of the relative dispersion, Eq. (4) can be written as

$\varepsilon^{2}=\frac{\int_{0}^{\infty}\left(r-r_{1}\right)^{2} n(r) \mathrm{d} r / N}{r_{1}^{2}}$,

where $r$ is the radius of the cloud droplet, $n(r)$ is the cloud droplet number concentration per unit of volume and per unit of radius, and $N$ is the total cloud droplet number concentration in a unit volume.

Equation (5) is then expanded to yield

$r_{1}^{2} \varepsilon^{2}=\frac{\int_{0}^{\infty}\left(r^{2}-2 r r_{1}+r_{1}^{2}\right) n(r) \mathrm{d} r}{N}$.

According to the definition of the average radius and the squared radius $\left(r_{2}\right)$, Eq. (6) can be written as

$r_{1}^{2} \varepsilon^{2}=r_{2}^{2}-2 r_{1}^{2}+r_{1}^{2}$

and Eq. (7) can be written as

$r_{2}^{2}=r_{1}^{2}\left(1+\varepsilon^{2}\right)$.

The skewness of the cloud droplet spectrum $(\alpha)$ is given by (Martin et al. 1994)

$\alpha=\frac{\int_{0}^{\infty}\left(r-r_{1}\right)^{3} n(r) \mathrm{d} r}{N \sigma^{3}}$.

The skewness represents the asymmetrical extent of the cloud droplet spectrum distribution relative to the average radius. At skewness of 0 , the cloud droplets spectral distribution relative to the average radius is completely symmetrical. 
According to the definition of skewness, Eq. (9) is changed to

$\alpha \sigma^{3}=\frac{\int_{0}^{\infty}\left(r^{3}-3 r^{2} r_{1}+3 r r_{1}^{2}-r_{1}^{3}\right) n(r) \mathrm{d} r}{N}$.

Moreover, using the definitions of the volume, square and average radius, Eq. (10) becomes

$\alpha \sigma^{3}=r_{3}^{3}-3 r_{2}^{2} r_{1}+3 r_{1}^{3}-r_{1}^{3}$.

Using Eq. (8), Eq. (11) is then transformed into

$r_{3}^{3}=r_{1}^{3}\left(1+3 \varepsilon^{2}+\alpha \varepsilon^{3}\right)$.

Equation (12) indicates that relative dispersion can be estimated from the average and volume radius of the cloud droplet if an accurate skewness value can be obtained. Previous work by Liu and Li (2015) showed that in the two-parameter cloud microphysics scheme, the average radius of cloud droplets can be obtained from the cloud droplets condensation equation and is given by the following:

$r_{1}=\frac{\mathrm{d} L_{w}}{\mathrm{~d} t} /\left(4 \pi \rho_{\mathrm{w}} N \frac{S}{G}\right)$,

where $L_{\mathrm{w}}$ is the liquid water content, $S$ the supersaturation of water vapor, $\rho_{\mathrm{w}}$ is the density of water, and $G$ is given by

$G=\left(\frac{L_{\mathrm{v}}}{R_{\mathrm{v}} T}-1\right) \frac{L_{\mathrm{v}} \rho_{\mathrm{w}}}{K_{\mathrm{d}} T}+\frac{\rho_{\mathrm{v}} R_{\mathrm{v}} T}{D_{\mathrm{v}} e_{\mathrm{s}}}$,

where $T$ is the temperature, $D_{\mathrm{v}}$ the water vapor diffusion coefficient, $K_{\mathrm{d}}$ the thermal conductivity, $L_{\mathrm{v}}$ the latent heat of condensation, $R_{\mathrm{v}}$ the water vapor gas constant, and $e_{\mathrm{S}}$ the saturation vapor pressure. For cloud droplets $(r>1 \mu \mathrm{m})$, Kelvin and Raoult effect can be ignored and the effects of ventilation and accommodating are also neglected. The volume radius of cloud droplets is given by

$r_{3}=\left(\frac{3}{4 \pi \rho_{\mathrm{w}}}\right)^{1 / 3}\left(\frac{L_{\mathrm{w}}}{N}\right)^{1 / 3}$.

Therefore, in the two-parameter cloud microphysics parameterization scheme and if the skewness is known, we can use Eq. (12) to find the cloud droplet spectrum relative dispersion without knowing or assuming the spectral distribution of cloud droplets.

\subsection{Approximation of zero skewness}

Equation (12) can be transformed into

$\varepsilon=\sqrt{\frac{1}{(3+\alpha \varepsilon)}\left(\frac{r_{3}^{3}}{r_{1}^{3}}-1\right)}$.
If the term containing the skewness is neglected in Eq. (16) or the skewness is assumed to be zero, the relative dispersion $(\tilde{\varepsilon})$ is approximated by

$\tilde{\varepsilon} \approx \sqrt{\frac{1}{3}\left(\frac{r_{3}^{3}}{r_{1}^{3}}-1\right)}$.

This approximation has been applied in some studies (Pontikis and Hicks 1992; Martin et al. 1994, Liu et al. 2006b). Martin et al. (1994) obtained the skewness of the cloud droplet spectrum distribution in continent and marine regions, respectively, and showed that the skewness of the cloud droplet spectrum mostly varied between -1 and +1 . They considered the skewness to be negligible and therefore neglected the term containing the skewness. So far, few studies have focused on the skewness of the cloud droplet spectrum. For this reason, we first investigate the skewness distributions of the cloud droplet spectrum.

According to the RACORO cloud data, the clouds are divided into two categories - the cumulus and the stratocumulus or stratus clouds. Figure 1a depicts a skewness distribution of cumulus clouds, showing that the skewness distribution is asymmetric with most skewness values greater than zero. The peak of the skewness distributions corresponds to a skewness of approximately 0.2 . The skewness ranges from -2.0 to +5 , with $99 \%$ of the data distributed between -1.0 and +2.0 , with an average skewness of 0.279 . The skewness distribution of stratocumulus and stratus clouds (Fig. 1b) again exhibits an obviously asymmetric distribution with the skewness values varying from -2.0 to +5 . The peak of the skewness distribution corresponds to a skewness of 0.2 , and most of the skewness values are clearly greater than zero with $99 \%$ of them falling between -1.5 and +3.5 and an average skewness of 0.63. A closer examination of the skewness data shows that high skewness values are typically found at the edge of the cloud with lower cloud water content. Although Fig. 1 shows that the skewness of the cloud droplet spectrum clusters is nearly zero, some high values of skewness are still present. This means that an assumption of zero skewness is not always valid and that the neglect of the terms containing the skewness is not well justified.

The ratio of estimated relative dispersion of the cloud droplet spectrum (Eq. 17) to its theoretical value (Eq. 16) is given by

$\frac{\tilde{\varepsilon}}{\varepsilon}=\sqrt{\frac{(3+\alpha \varepsilon)}{3} .}$

This ratio measures the difference between the estimated relative dispersion and its theoretical value. A ratio of one indicates that the two values are equal. Inspection of the 
Fig. 1 Skewness of cloud droplet spectrum: a cumulus and $\mathbf{b}$ stratocumulus and stratus

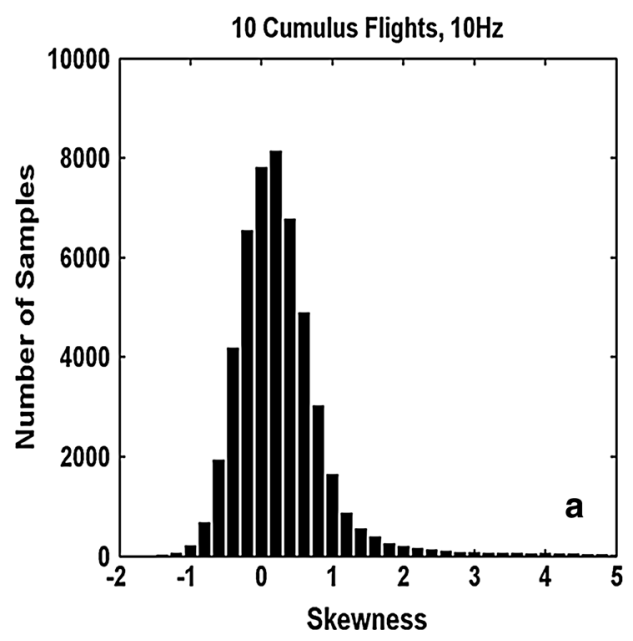

right-hand side of Eq. (17) shows that the deviation of the estimate from the theoretical value is related to skewness and to the product of the skewness and the relative dispersion $(\alpha \varepsilon)$. Moreover, when $\alpha \varepsilon \geq 0, \tilde{\varepsilon} \geq \varepsilon$, and when $\alpha \varepsilon \leq 0, \tilde{\varepsilon} \leq \varepsilon$. If a relative error of less than $15 \%$ for the estimate is assumed to be acceptable, from Eq. (18) we obtain

$0.85 \leq \sqrt{\frac{3+\alpha \varepsilon}{3}} \leq 1.15$

and

$-0.8325 \leq \alpha \varepsilon \leq 0.9675$.

Since its values correspond to the maximums of permissible relative deviation, the product $\alpha \varepsilon$ is thus very important for the accuracy of the estimation. Next, we analyze the $\alpha \varepsilon$ distribution based on the observational data.

Figure 2a shows the distribution of the product of skewness and relative dispersion $(\alpha \varepsilon)$ for the cumulus clouds, which is asymmetric with most of the values greater than zero. The $\alpha \varepsilon$ peak corresponds to zero $\alpha \varepsilon$. The

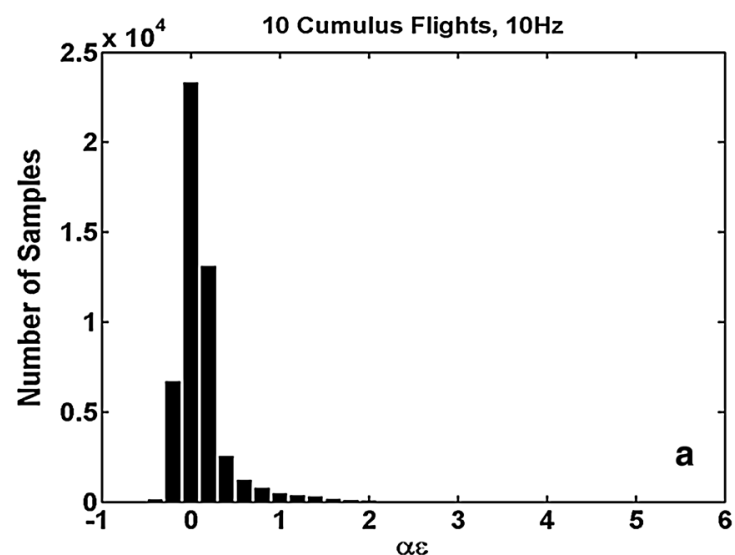

values of $\alpha \varepsilon$ range from -1.0 to +5 with $97.2 \%$ of the data distributed between -0.84 and +0.96 , satisfying the accuracy criterion for estimates (i.e., the relative deviation lesser than $15 \%$ ). Figure $2 b$ depicts the $\alpha \varepsilon$ distribution for the stratocumulus and stratus. Again, the $\alpha \varepsilon$ distribution is asymmetric, with a peak $\alpha \varepsilon$ also corresponding to zero $\alpha \varepsilon$ and most of the data obviously greater than zero. The $\alpha \varepsilon$ values range from -1.0 to +5 with $87.3 \%$ of the data distributed approximately between -0.84 and +0.96 , guaranteeing a relative deviation smaller than $15 \%$ for the estimates. A comparison of Fig. 2a, b indicates that the $\alpha \varepsilon$ distribution for cumulus clouds is more concentrated near zero, and its relative deviation for the estimates obtained from Eq. (16) is smaller than that for the stratocumulus and stratus data.

\subsection{Approximation method of the gamma distribution}

A large number of observations have shown that a threeparameter gamma function can well describe the cloud

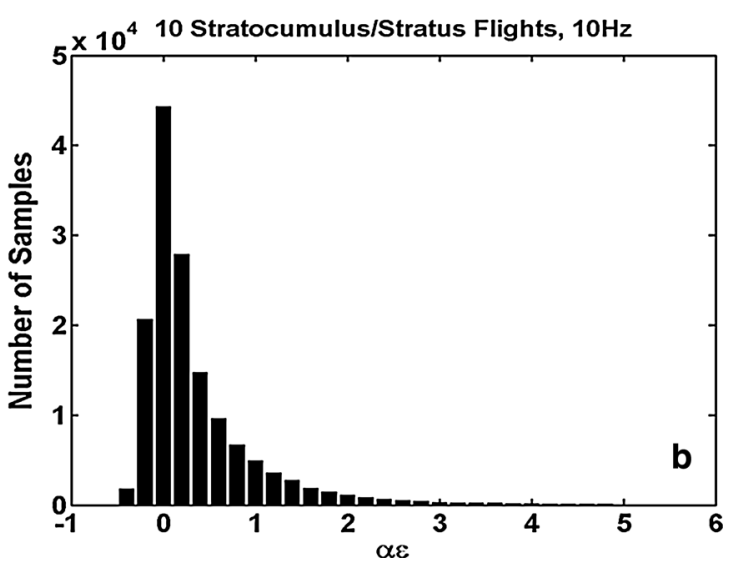

Fig. 2 Product of skewness and relative dispersion of cloud droplet spectrum for a cumulus and $\mathbf{b}$ stratocumulus and stratus 
droplet spectrum distribution (Mason 1971). For such a distribution, the $p$ th moment of the cloud droplet radius $\left(M_{\mathrm{p}}\right)$ is given by

$M_{\mathrm{p}}=\left(\frac{1}{\lambda}\right)^{\mathrm{p}} \frac{\Gamma(\mu+p+1)}{\Gamma(\mu+1)}$,

where $\Gamma$ is the gamma function and $p$ is the order. Therefore, Eq. (12) becomes

$\frac{r_{3}^{3}}{r_{1}^{3}}=\frac{(\mu+3)(\mu+2)}{(\mu+1)^{2}}=1+3 \varepsilon^{2}+\alpha \varepsilon^{3}$,

and for the gamma distribution, $\varepsilon^{2}=\frac{1}{\mu+1}$. From Eq. (22), we obtain that

$\alpha=2 \varepsilon$.

Equation (23) indicates that the presence of the gamma distribution is similar to assuming that the skewness is equal to twice the relative dispersion. The percentage at which the gamma distribution approximation satisfies the condition of the relative standard deviation less than $15 \%$ is $99.3 \%$ for cumulus and $98.7 \%$ for stratus and stratocumulus. Therefore, the use of gamma distribution for obtaining $\varepsilon$ is better than an assumption of zero skewness, especially for stratocumulus and stratus.

\subsection{New estimation method}

The above analysis with regard to gamma distribution indicates that the gamma distribution is similar to the assumption that the skewness is two times that of relative dispersion. A close examination of the scatter plot of observed skewness versus $\varepsilon$ (including cumulus, and stratocumulus and stratus) presented in Fig. 3 shows that the relationship between skewness and relative dispersion is not simply linear and that the skewness is also affected by volume radius and average radius. Thus, the assumption

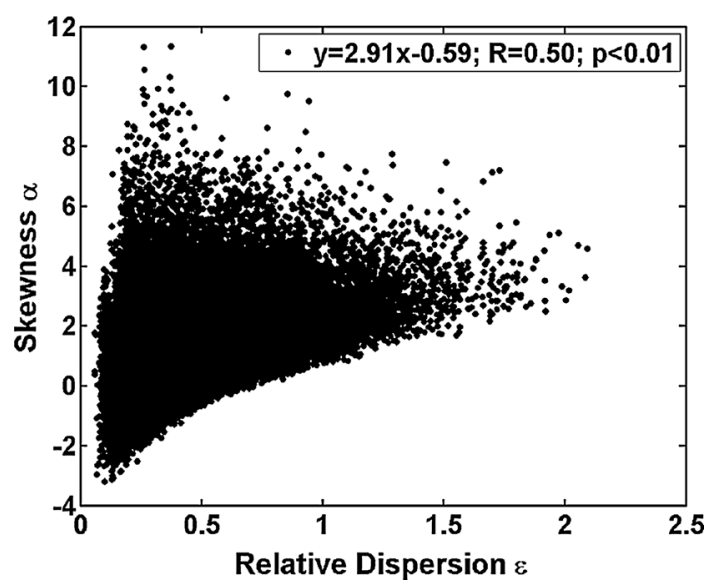

Fig. 3 Scatter plot of the observed skewness versus $\varepsilon$ that the skewness is equal to twice the relative dispersion does not yield the best linear fit to the data. Using the least square method, we obtain the best linear fit as follows:

$\alpha=2.91 \varepsilon-0.59$.

Substituting Eq. (24) into Eq. (12), we obtain

$r_{3}^{3}=r_{1}^{3}\left(1+3 \varepsilon^{2}-0.59 \varepsilon^{3}+2.91 \varepsilon^{4}\right)$.

The relative dispersion can be obtained from Eq. (25). However, this approach can be complicated because we need to first solve a cubic equation and then two quadratic equations based on the method of solving quartic equations. We instead construct an iterative algorithm based on Eqs. (16) and (24) to obtain the relative dispersion:

1. Assuming $\alpha=0$, then

$$
\varepsilon_{1}=\sqrt{\frac{1}{3}\left(\frac{r_{3}^{3}}{r_{1}^{3}}-1\right)} .
$$

2. Iterating, $i=1,2, \ldots N$,

$$
\begin{aligned}
& \alpha_{i}=2.91 \varepsilon_{i}-0.59 \\
& \varepsilon_{i+1}=\sqrt{\frac{1}{\left(3+\alpha_{i} \varepsilon_{i}\right)}\left(\frac{r_{3}^{3}}{r_{1}^{3}}-1\right)} .
\end{aligned}
$$

This iteration ends when $-0.001 \leq \frac{\varepsilon_{i+1}-\varepsilon_{i}}{\varepsilon_{i}} \leq 0.001$.

\section{Test and comparison of three methods}

To further evaluate the three different methods described in Sect. 3, we calculate the relative dispersion using all of them and compare the estimates with the observed ones (based on the definition and data of the cloud droplet spectrum). Figure 4 shows the scatter plots of the observed versus the calculated cumulus relative dispersion. As demonstrated in Fig. 4a, the deviation for the results obtained with the first method (zero skewness) is small at the lower end of relative dispersion $(<1.0)$ and then increases rapidly at the higher end $(>1.0)$. However, only a small fraction of data, approximately $0.073 \%$, exceeds 1.0 and the correlation coefficient between the estimates and the observations is 0.98 . The relative dispersions values resulted from the second method (assuming that the gamma distribution) are well correlated with the observation data (Fig. 4b) with a correlation coefficient of 0.99 . In the case of relative dispersion greater than one, the deviation is noticeably smaller than that produced by the first method. However, it can still be clear that most of the values obtained with the second method are higher than the observed values. Estimates of relative dispersion from our proposed new method are evaluated in Fig. 4c. The 

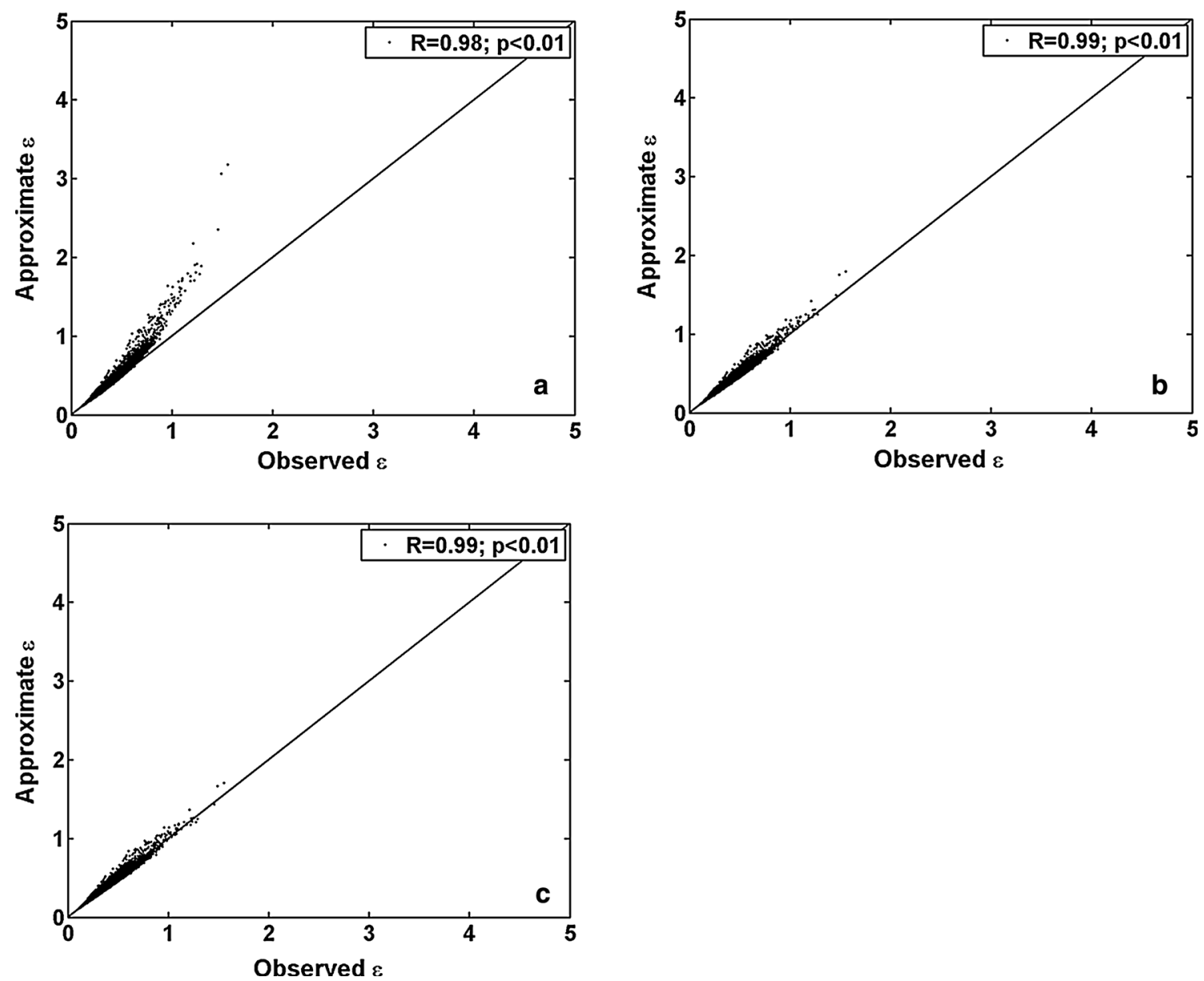

Fig. 4 Scatter plot of the relative dispersion for cumulus between observations and three estimation methods: a skewness of zero, b gamma distribution and $\mathbf{c}$ new method

estimates and the observation are in very good correlation with the correlation coefficient, reaching 0.99 . For the relative dispersion values higher than one, the new method is slightly better than the second method and shows smaller deviations from the observed values.

Figure 5 shows the comparisons of the estimated and observed relative dispersion data for the stratocumulus and stratus. Figure 5a shows that the results from the first method for stratocumulus and stratus deviates slightly for relative dispersion values below 1.0, but the deviation increases quickly for the case of relative dispersion values above 1.0. The faction of relative dispersion values higher than one is approximately $1.6 \%$. This fraction is approximately 22 times higher than that of cumulus, and the deviation increased significantly. The relative dispersion values obtained using the second method show good correlation with the observation data with a correlation coefficient of 0.99 (Fig. 5b). For the relative dispersion values greater than one, the deviation is obviously smaller than that of the first method. It is found that most of the values obtained with the second method are slightly higher than the corresponding observation values. Figure 5c presents the scatter plot of relative dispersion values obtained with the new method versus observations, showing that the estimates are in close agreement with the observations with a correlation coefficient of 0.99 . As compared to the second method, it is found that the values obtained with the new method are distributed symmetrically relative to the observations. The estimated values from the new method are slightly smaller than the observed values for the relative dispersion greater than one, but these deviations are reduced in comparison to those resulting from the second method.

Table 1 lists the standard errors of the three methods. Standard errors of three methods for cumulus clouds are all less than those for the stratus and stratocumulus. The standard errors of the new method are the least among the three methods. The standard errors from the second method are marginally greater than those of the new method. The results from this error analysis indicate that the new method has the highest precision among the three methods. 

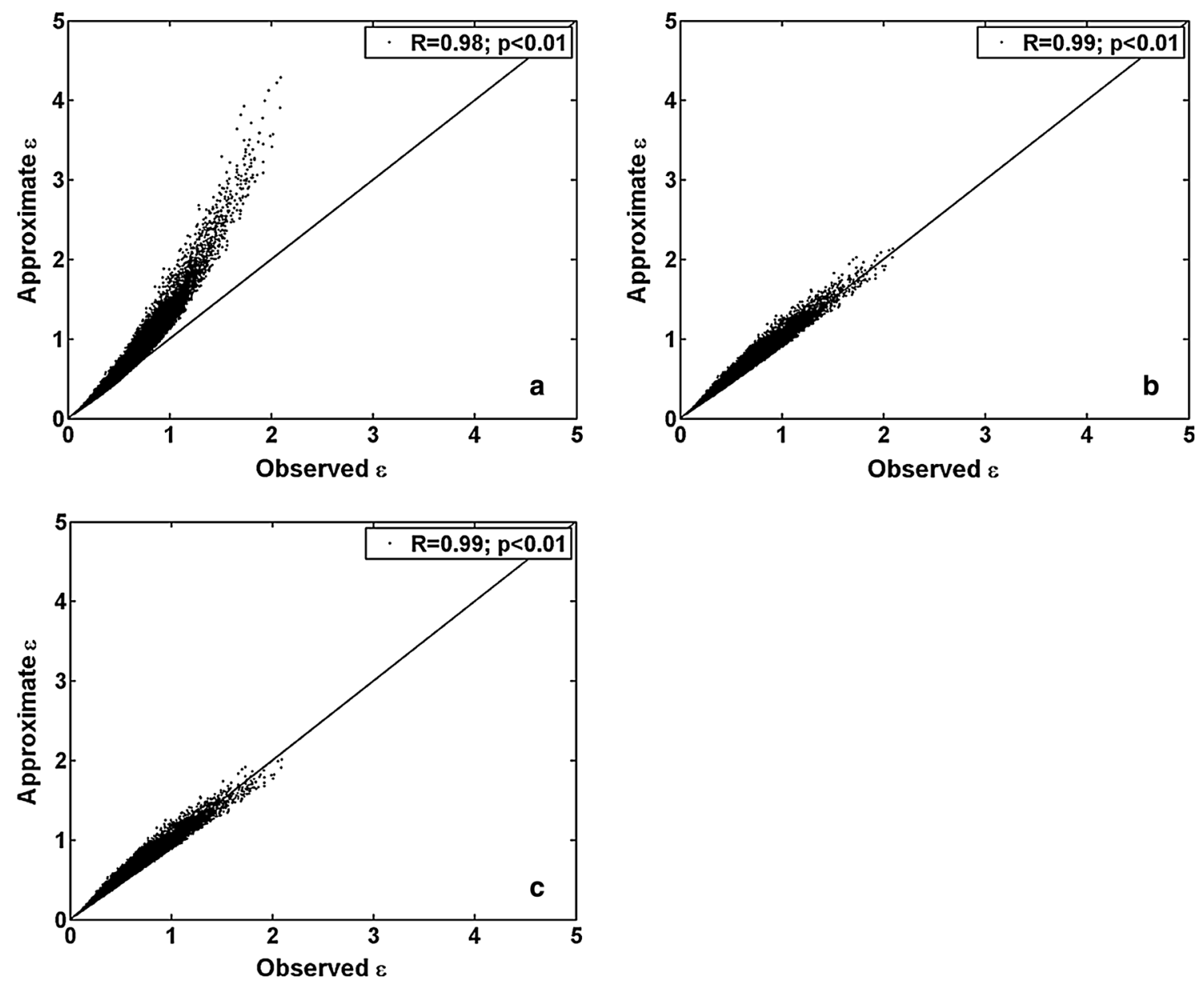

Fig. 5 Scatter plot of relative dispersion for stratocumulus and stratus between observations and three estimation methods: a skewness of zero, b gamma distribution and $\mathbf{c}$ new method

Table 1 Standard error of the three methods for the relative dispersion

Table 2 Standard error of the three methods for the effective radius (unit: $\mu \mathrm{m}$ )

\begin{tabular}{llll}
\hline & Zero skewness & Gamma distribution & New method \\
\hline Cumulus & 0.0336 & 0.0176 & 0.0155 \\
Stratocumulus and stratus & 0.1026 & 0.0261 & 0.0245 \\
\hline
\end{tabular}

\begin{tabular}{llll}
\hline & Zero skewness & Gamma distribution & New method \\
\hline Cumulus & 0.1165 & 0.0624 & 0.0534 \\
Stratocumulus and stratus & 0.4294 & 0.1598 & 0.1605 \\
\hline
\end{tabular}

In addition, the relationship between the skewness and the relative dispersion (Eq. 24) can also be directly used to solve the cloud effective radius. According to Eqs. (8) and (12), the effective radius $\left(r_{\mathrm{e}}\right)$ is

$$
r_{\mathrm{e}}=\frac{r_{3}^{3}}{r_{2}^{2}}=\frac{\left(1+3 \varepsilon^{2}+\alpha \varepsilon^{3}\right)^{2 / 3}}{1+\varepsilon^{2}} r_{3} .
$$

Using the observed data, the volume radius, square radius, and relative dispersion are calculated. Next, following the definition of the effective radius and Eqs. (24) and (29), the observed and estimated effective radii are obtained, respectively. The estimates are well correlated with the observations (figure omitted). The correlation coefficients are close to one except for the case of stratocumulus and stratus using the first method, whose correlation coefficient reaches 0.99 . Similar to Table 1, Table 2 is the standard error of the effective radius between the estimates and the observations, from which the new 
method can be seen to produce the smallest standard error for cumulus clouds. In stratus and stratocumulus, the deviation of the new method is closely comparable to that of gamma distribution. Overall, the new method is the best.

\section{Summary}

First, the above analyses reveal that the skewness distribution of cloud droplet spectra is asymmetric for both cumulus and stratocumulus and stratus, especially for the latter. Second, relative dispersion of the cloud droplet spectrum is very important for cloud microphysical parameterization. An assumption of zero skewness is shown to produce relatively large deviations between the estimates and the observations. Moreover, if the term containing skewness is omitted in calculating relative dispersion, its deviations from the observations are related not only to the skewness, but also to the product of the relative dispersion and skewness. Third, the gamma distribution is similar to assume that skewness is twice the relative dispersion. The relative dispersion derived under the assumption of gamma distribution is better than that assuming zero skewness. As shown above, the new method (Eq. 25) to estimate the relative dispersion is developed using the best linear fitting (Eq. 24) between skewness and relative dispersion of the observational data. This new method does not depend on the droplet spectrum distribution assumption and hence widens it applicability. Comparisons with the observation show that the new method is better than that assuming the gamma distribution and is the best among all three methods. Finally, standard error analyses and the new method establishment illustrate that, if a better correlation between skewness and relative dispersion could be found, estimation deviations of the relative dispersion from the observations would be reduced according to Eq. (12). Recognizing that this work only involved those data from the RACORO experiment, we recommend that more observations should be analyzed to test the applicable scope of the new method.

Acknowledgments This work was supported by the National Basic Research Program of China (Grant No. 2011CB403406) and the National Natural Science Foundation of China (91537213, 91537108, 41305120). We thank the ARM project team for providing the observational data of the RACORO experiment.

Open Access This article is distributed under the terms of the Creative Commons Attribution 4.0 International License (http://crea tivecommons.org/licenses/by/4.0/), which permits unrestricted use, distribution, and reproduction in any medium, provided you give appropriate credit to the original author(s) and the source, provide a link to the Creative Commons license, and indicate if changes were made.

\section{References}

Bohren CF (1987) Multiple scattering of light and some of its observable consequences. Am J Phys 55:524-533

Cohard JM, Pinty JP (2000) A comprehensive two-moment warm microphysical bulk scheme. I: description and tests. Q J R Meteor Soc 126:1815-1842. doi:10.1002/qj.49712656613

Cotton WR, Anthes RA (1989) Storm and cloud dynamics. Academic Press, London

Deng Z, Zhao C, Zhang Q, Huang M, Ma X (2009) Statistical analysis of microphysical properties and the parameterization of effective radius of warm clouds in Beijing area. Atmos Res 93:888-896. doi:10.1016/j.atmosres.2009.04.011

Ferrier BS (1994) A double-moment multiple-phase four-class bulk ice scheme. Part I: description. J Atmos Sci 51:249-280. doi:10. 1175/1520-0469(1994)051<0249:ADMMPF > 2.0.CO;2

Hudson JG, Yum SS (1997) Droplet spectral broadening in marine stratus. J Atmos Sci 54:2642-2654. doi:10.1175/15200469(1997)054<2642:dsbims >2.0.co;2

Khvorostyanov VI, Curry JA (1999a) Toward the theory of stochastic condensation in clouds. Part I: a general kinetic equation. J Atmos Sci 56:3985-3996. doi:10.1175/1520-0469(1999)056< 3985:TTTOSC $>2.0 . \mathrm{CO} ; 2$

Khvorostyanov VI, Curry JA (1999b) Toward the theory of stochastic condensation in clouds. Part II: analytical solutions of the gamma-distribution type. J Atmos Sci 56:3997-4013. doi:10. 1175/1520-0469(1999)056<3997:TTTOSC $>2.0 . C O ; 2$

Kogan YL, Belochitski A (2012) Parameterization of cloud microphysics based on full integral moments. J Atmos Sci 69:2229-2242. doi:10.1175/JAS-D-11-0268.1

Liu YG, Daum PH (2000) Spectral dispersion of cloud droplet size distributions and the parameterization of cloud droplet effective radius. Geophys Res Lett 27:1903-1906. doi:10.1029/ 1999GL011011

Liu YG, Daum PH (2002) Anthropogenic aerosols: indirect warming effect from dispersion forcing. Nature 419:580-581. doi:10.1002/ (SICI)1097-0177(199909)216:1<1:AID-DVDY1>3.0.CO;2-T

Liu Y, Li W (2015) A method for solving relative dispersion of the cloud droplet spectra. Sci China Earth Sci 58:929-938. doi:10. 1007/s11430-015-5059-9

Liu YG, Daum PH, McGraw R, Miller M (2006a) Generalized threshold function accounting for effect of relative dispersion on threshold behavior of autoconversion process. Geophys Res Lett 33:L11804. doi:10.1029/2005GL025500

Liu YG, Daum PH, Yum SS (2006b) Analytical expression for the relative dispersion of the cloud droplet size distribution. Geophys Res Lett 33:L02810. doi:10.1029/2005GL024052

Lu ML, Conant WC, Jonsson HH, Varutbangkul V, Flagan RC, Seinfeld JH (2007) The marine stratus/stratocumulus experiment (MASE): aerosol-cloud relationship in marine stratocumulus. J Geophys Res 112:D10209. doi:10.1029/2006JD007985

Lu CS, Liu YG, Niu SJ, Vogelmann AM (2012) Observed impacts of vertical velocity on cloud microphysics and implications for aerosol indirect effects. Geophys Res Lett 39:L21808. doi:10. 1029/2012GL053599

Lu CS, Liu YG, Niu SJ, Vogelmann AM (2013) Empirical relationship between entrainment rate and microphysics in cumulus clouds. Geophys Res Lett 40:2333-2338. doi:10.1002/grl.50445

Lu CS, Liu YG, Zhang GJ, Wu XH, Endo S, Cao L, Li YQ, Guo XH (2016) Improving parameterization of entrainment rate for shallow convection with aircraft measurements and large-eddy simulation. J Atmos Sci 73:761-773. doi:10.1175/JAS-D-150050.1 
Ma J, Chen Y, Wang W, Yan P, Liu H, Yang S, Hu Z, Lelieveld J (2010) Strong air pollution causes widespread haze-clouds over China. J Geophys Res 115:D18204. doi:10.1029/2009JD013065

Martin GM, Johnson DW, Spice A (1994) The measurement and parameterization of effective radius of droplets in warm stratocumulus clouds. J Atmos Sci 51:1823-1842. doi:10.1175/ 1520-0469(1994)051<1823:TMAPOE $>2.0 . \mathrm{CO} ; 2$

Mason BJ (1971) Physics of clouds. Clarendon Press, Oxford

Meyers MP, Walko RL, Harrington JY, Cotton WR (1997) New RAMS cloud microphysics parameterization. Part II: the twomoment scheme. Atmos Res 45:3-39. doi:10.1016/S01698095(97)00018-5

Milbrandt JA, Yau MK (2005a) A multimoment bulk microphysics parameterization. Part I: analysis of the role of the spectral shape parameter. J Atmos Sci 62:3051-3064. doi:10.1175/JAS3534.1

Milbrandt JA, Yau MK (2005b) A multimoment bulk microphysics parameterization. Part II: a proposed three-moment closure and scheme description. J Atmos Sci 62:3065-3081. doi:10.1175/ JAS3535.1

Miles NL, Verlinde J, Clothiaux EE (2000) Cloud droplet size distributions in low-level stratiform clouds. J Atmos Sci 57:295-311. doi:10.1175/1520-0469(2000)057<0295:CDSDIL> 2.0.CO;2

Morrison H, Curry JA, Shupe MD, Zuidema P (2005) A new doublemoment microphysics parameterization for application in cloud and climate models. Part II: single-column modeling of arctic clouds. J Atmos Sci 62:1678-1693. doi:10.1175/JAS3447.1

Pawlowska H, Grabowski WW, Brenguier JL (2006) Observations of the width of cloud droplet spectra in stratocumulus. Geophys Res Lett 33:L19810. doi:10.1029/2006GL026841
Pontikis C, Hicks E (1992) Contribution to the cloud droplet effective radius parameterization. Geophys Res Lett 19:2227-2230. doi:10.1029/92GL02283

Pruppacher HR, Klett JD (1997) Microphysics of clouds and precipitation. Springer, Berlin

Rossow WB, Schiffer RA (1999) Advances in understanding clouds from ISCCP. Bull Am Meteorol Soc 80:2261-2287. doi:10. 1175/1520-0477(1999)080<2261:AIUCFI >2.0.CO;2

Sedunov Y (1974) Physics of drop formation in the atmosphere. Wiley, Oxford

Vogelmann AM, McFarquhar GM, Ogren JA, Turner DD, Comstock JM, Feingold G, Long CN, Jonsson HH, Bucholtz A, Collins DR, Diskin GS, Gerber H, Lawson RP, Woods RK, Andrews E, Yang HJ, Chiu JC, Hartsock D, Hubbe JM, Lo C, Marshak A, Monroe JW, McFarlane SA, Schmid B, Tomlinson JM, Toto T (2012) Racoro extended-term aircraft observations of boundary layer clouds. Bull Am Meteorol Soc 93:861-878. doi:10.1175/BAMS-D-11-00189.1

Xie X, Liu X, Peng Y, Wang Y, Yue Z, Li X (2013) Numerical simulation of clouds and precipitation depending on different relationships between aerosol and cloud droplet spectral dispersion. Tellus B 65:19054. doi:10.3402/tellusb.v65i0.19054

Zhao CS, Tie XX, Brasseur G, Noone KJ, Nakajima T, Zhang Q, Zhang RY, Huang MY, Duan Y, Li GL, Ishizaka Y (2006) Aircraft measurements of cloud droplet spectral dispersion and implications for indirect aerosol radiative forcing. Geophys Res Lett 33:L16809. doi:10.1029/2006GL02665 